\title{
SEPARATION IN COUNTABLY PARACOMPACT SPACES
}

\author{
BY
}

W. STEPHEN WATSON

\begin{abstract}
We study the question "Are discrete families of points separated in countably paracompact spaces?" in the class of first countable spaces and the class of separable spaces.
\end{abstract}

Two of the main directions of research in general topology in the last thirty years have been the work of Jones, Bing, Tall, Fleissner, Nyikos and others motivated by the normal Moore space problem (when are discrete families separated in normal spaces?) and the work of Rudin, Zenor and others motivated by the Dowker space problem (what is the relation between normal and countably paracompact?). This paper considers the following question:

(A) Are discrete families of points separated in countably paracompact spaces?

There is a related question:

(B) Are discrete families of points separated in normal spaces?

This question has been studied in the class of first countable spaces and in the class of separable spaces (and so that is also where we consider question (A)) and has been more or less answered in those classes:

In the class of first countable spaces, question (B) is independent of the axioms of set theory: $V=L$ and other axioms imply yes (Fleissner, Tall $[\mathbf{1}, \mathbf{1 1}])$ and $\mathrm{MA}+\neg \mathrm{CH}$ implies no (Silver, Rothberger, Bing, Tall [11]). In the class of separable spaces, (B) is equivalent to the cardinal arithmetic $2^{\aleph_{0}}<2^{\aleph_{1}}$ (Jones, Heath [7, 5]).

The negative results consist of the construction of counterexamples which are countably paracompact (so that these negative results also apply to (A)) while the positive results consist of proofs for which normality appears essential. Fleissner [3] and Tall have asked whether it is consistent that, in the class of first countable spaces, the answer is yes to (A). Fleissner, Przymusinski and Reed [3, 8, 9] have asked whether it is possible to show that, in the class of separable spaces, (A) is equivalent to the cardinal arithmetic $2^{\aleph_{0}}<2^{\aleph_{1}}$ (Fleissner was able to extend Jones' short proof to show that, in the class of separable spaces, $2^{\aleph_{0}}=\aleph_{1}$ implies yes to (A)). We show that, in the class of first countable spaces, $(\mathrm{A})$ is independent of the axioms of set theory ( $V=L$ implies yes) and that, in the class of separable spaces, (A) is equivalent to a set-theoretic statement whose equivalence with $2^{\aleph_{0}}<2^{\aleph_{1}}$ is a special case of a well-known open problem in set theory (Steprans, Jech and Prikry $[6,10]$ have shown, independently, for example, that, if $2^{\aleph_{0}}$ is a regular cardinal and there is no measurable cardinal in an inner model, then the equivalence holds).

In this paper, a space is a regular topological space; a family $\left\{A_{\alpha}: \alpha<\kappa\right\}$ of subsets of a space is separated if there is a disjoint family $\left\{O_{\alpha}: \alpha<\kappa\right\}$ of open sets

Received by the editors July 9, 1982 and, in revised form, November 15, 1984.

1980 Mathematics Subject Classification. Primary 54D15; Secondary 54D18, 54A35.

(C) 1985 American Mathematical Society $0002-9947 / 85 \$ 1.00+\$ .25$ per page 
such that, for each $\alpha<\kappa, A_{\alpha}$ is contained in $O_{\alpha}$; a space is collectionwise Hausdorff if every discrete family of points is separated.

\section{First countable countably paracompact spaces.}

THEOREM $1(V=L)$. First countable countably paracompact spaces are collectionwise Hausdorff.

To motivate the method of proof of this result, we discuss the proof (due to Fleissner and Tall) that it is consistent that first countable normal spaces are collectionwise Hausdorff.

In 1969, Tall [11] showed that, in an iterated forcing extension, first countable normal spaces of cardinality less than $\aleph_{\omega_{1}}$ are collectionwise Hausdorff. In 1972, Fleissner [1] showed that, under $V=L$, first countable normal spaces are collectionwise Hausdorff.

It seems to be necessary to treat regular and singular cardinals differently $\left(\aleph_{\omega_{1}}\right.$ is the least singular cardinal of uncountable cofinality) and Fleissner's result follows, by induction on $\kappa$, from:

LEMMA $1(V=L)$. Let $X$ be a normal space and let $\kappa$ be a cardinal such that each discrete family of points of cardinality less than $\kappa$ is separated. If (a) $\kappa$ is regular and the character of $X$ is at most $\kappa$, or if (b) $\kappa$ is singular and the character of $X$ is less than $\kappa$, then each discrete family of points of cardinality $\kappa$ is separated.

The induction step for regular cardinals uses a combinatorial consequence of $V=L$ (an enumeration principle) which allows, assuming the existence of a discrete family of points of cardinality $\kappa$ which is not separated, the inductive definition of a partition (a counterexample to normality) of the discrete family of points by witnessing the failure of each neighborhood assignment to separate the family. There are $2^{\kappa}$ many neighborhood assignments which must be witnessed in $\kappa$ steps and that is the use of $V=L$.

The induction step for singular cardinals is complicated by the lack of useful enumeration principles for singular cardinals which are stronger than the generalized continuum hypothesis. The GCH allows the inductive definition of a counterexample to normality but by witnessing the failure of each neighborhood assignment to separate "most" of the discrete family of points. Iterating countably many times the process of separating "most" of the remaining unseparated subfamilies yields a countable partition of the family into separated subfamilies so that the family is separated by applying normality.

The proof of Theorem 1 is similar, in outline, to the proof of Fleissner's result. Theorem 1 follows, by induction on $\kappa$, from the following lemma.

LEMMA $2(V=L)$. Let $X$ be a countably paracompact space and let $\kappa$ be a cardinal such that each discrete family of points of cardinality less than $\kappa$ is separated. If (a) $\kappa$ is regular and the character of $X$ is most most $\kappa$, or if (b) $\kappa$ is singular and the character of $X$ is less than $\kappa$, then each discrete family of points of cardinality $\kappa$ is separated.

Let us fix some notation throughout this section: $X$ is a countably paracompact space with character $\lambda$. $\left\{x_{\alpha}: \alpha \in \kappa\right\}$ is a discrete family of points and, for each $\alpha \in \kappa,\left\{U_{\beta}(\alpha): \beta \in \lambda\right\}$ is a neighborhood base for $x_{\alpha}$. 
We begin with an application of countable paracompactness:

LEMMA 3. Whenever $m: \kappa \rightarrow \omega$, there is $g: \kappa \rightarrow \lambda$ such that, letting $j: \kappa \rightarrow$ $[\omega]^{<\omega}$ be defined by $j(\alpha)=\left\{m(\beta): U_{g(\alpha)}(\alpha) \cap U_{g(\beta)}(\beta) \neq \emptyset\right\},\left\{j(\beta): U_{g(\alpha)}(\alpha) \cap\right.$ $\left.U_{g(\beta)}(\beta) \neq \emptyset\right\}$ is finite.

Proof. For each $n \in \omega$, let $A_{n}=\left\{x_{\alpha}: m(\alpha)=n\right\} .\left\{A_{n}: n \in \omega\right\}$ is a partition of $\left\{x_{\alpha}: \alpha \in \kappa\right\}$ into a countable discrete family of closed sets. Applying countable paracompactness, let $\left\{U_{n}: n \in \omega\right\}$ be a locally finite family of open sets such that $U_{n}$ contains $A_{n}$. Let $f_{0}$ witness the local finiteness of $\left\{U_{n}: n \in \omega\right\}$. That is, let $f_{0}: \kappa \rightarrow \lambda$ be defined such that, for each $\alpha \in \kappa$,

(1) $\left\{n \in \omega: U_{f_{0}(\alpha)}(\alpha) \cap U_{n}\right\}$ is finite.

Let $h$ be the "way" in which $\left\{U_{n}: n \in \omega\right\}$ is locally finite. That is, let $h: \kappa \rightarrow$ $[\omega]^{<\omega}$ be defined by $h(\alpha)=\left\{n \in \omega: U_{f_{0}(\alpha)}(\alpha) \cap U_{n} \neq \emptyset\right\}$.

For each $p \in[\omega]^{<\omega}$, let $B_{p}=\left\{x_{\alpha}: h(\alpha)=p\right\}$. $\left\{B_{p}: p \in[\omega]^{<\omega}\right\}$ is a partition of $\left\{x_{\alpha}: \alpha \in \kappa\right\}$ into a countable discrete family of closed sets. Applying countable paracompactness, let $\left\{V_{p}: p \in[\omega]^{<\omega}\right\}$ be a locally finite family of open sets such that $V_{p}$ contains $B_{p}$. Let $f_{1}$ witness the local finiteness of $\left\{V_{p}: p \in[\omega]^{<\omega}\right\}$. That is, let $f_{1}: \kappa \rightarrow \lambda$ be defined such that, for each $\alpha \in \kappa$,

(2) $\left\{p \in[\omega]^{<\omega}: U_{f_{1}(\alpha)}(\alpha) \cap V_{p} \neq \emptyset\right\}$ is finite.

Let $g: \kappa \rightarrow \lambda$ be defined such that, for each $\alpha \in \kappa, U_{g(\alpha)}(\alpha)$ is contained in each of $U_{m(\alpha)}, U_{f_{0}(\alpha)}(\alpha), V_{h(\alpha)}, U_{f_{1}(\alpha)}(\alpha)$. That is, let $g$ refine $\left\{U_{n}: n \in \omega\right\}$, witness the local finiteness of $\left\{U_{n}: n \in \omega\right\}$, refine the "way" in which $\left\{U_{n}: n \in \omega\right\}$ is locally finite and witness the local finiteness of the "way" in which $\left\{U_{n}: n \in \omega\right\}$ is locally finite.

We show that $g$ works. $U_{g(\alpha)}(\alpha) \cap U_{g(\beta)}(\beta) \neq \emptyset$ and $m(\beta)=n$ implies $U_{f_{0}(\alpha)}(\alpha) \cap$ $U_{n} \neq \emptyset$ (since $U_{g(\alpha)}(\alpha) \subset U_{f_{0}(\alpha)}(\alpha)$ and $\left.U_{g(\beta)}(\beta) \subset U_{m(\beta)}\right)$ and so, by $(1), j$ is well defined. $U_{g(\alpha)}(\alpha) \cap U_{g(\beta)}(\beta) \neq \emptyset$ and $h(\beta)=p$ implies $U_{f_{1}(\alpha)}(\alpha) \cap V_{p} \neq \emptyset$ (since $U_{g(\alpha)}(\alpha) \subset U_{f_{1}(\alpha)}(\alpha)$ and $\left.U_{g(\beta)}(\beta) \subset V_{h(\beta)}\right)$ and so, by $(2),\left\{h(\beta): U_{g(\alpha)}(\alpha) \cap\right.$ $\left.U_{g(\beta)}(\beta) \neq \emptyset\right\}$ is finite. $h$ does not coincide with $j$ but, for each $\beta \in \kappa, h(\beta)$ contains $j(\beta)$ (since $U_{g(\beta)}(\beta) \subset U_{f_{0}(\beta)}(\beta)$ ). There are finitely many subsets of each of the finitely many $h(\beta)$ implies that $\left\{j(\beta): U_{g(\alpha)}(\alpha) \cap U_{g(\beta)}(\beta) \neq \emptyset\right\}$ is finite.

The induction step for regular cardinals uses a combinatorial consequence of $V=L$ (an enumeration principle) which allows, assuming the existence of a discrete family of points of cardinality $\kappa$ which is not separated, the inductive definition of a countable partition (a counterexample to countable paracompactness) of the discrete family of points by witnessing the failure of each neighborhood assignment to both (a) separate the family and (b) witness the way in which the space is countably paracompact with respect to the partition. The enumeration principle "traps", in addition to neighborhood assignments, the way in which the space is countably paracompact with respect to the partition. The partition is defined inductively by mapping a point somewhere that the enumeration principle "guesses" it is not mapped. This is the main new set-theoretic idea of the proof.

The proof of Lemma 2(a) may appear technical so we shall first present a proof of the following lemma of Fleissner $[\mathbf{2}, \mathbf{4}]$ in which the ideas are more accessible.

LEMMA 4. If $\diamond_{S}$ is true for each stationary set $S$, then special Aronszajn trees are not countably paracompact. 
ProOF. Let $T$ be a special Aronszajn tree. $T$ is the union of countably many antichains. Some antichain $A$ must intersect a stationary set $S$ of levels. For $\alpha \in S$, let $T_{\alpha}$ be the $\alpha$ th level of $T$. For $\alpha \in S$, let $x_{\alpha} \in A \cap T_{\alpha}$. Let $U_{\alpha}(\beta)$ be $\left\{t \in T: t \leq x_{\beta}\right.$ and level $\left.(t) \geq \alpha\right\}$ when $\alpha<\beta$ and $T$ otherwise.

Let $\left\{\left(g_{\alpha}, j_{\alpha}\right): \alpha \in S\right\}$ be such that, for any $(g, j)$ where $g: S \rightarrow \omega_{1}$ and $j: S \rightarrow$ $[\omega]<\omega\left([\omega]^{<\omega}\right.$ denotes the family of finite subsets of $\left.\omega\right)$, there are stationarily many $\alpha \in S$ such that $g \uparrow \alpha=g_{\alpha}$ and $j \uparrow \alpha=j_{\alpha}$. $\diamond_{S}$ implies that this sequence exists! We define $m: S \rightarrow \omega$ as follows: If there is a neighborhood $V$ of $x_{\alpha}$ such that $J=\bigcup\left\{j_{\alpha}(\beta): U_{g_{\alpha}(\beta)}(\beta) \cap V \neq \emptyset\right.$ and $\left.\beta<\alpha\right\}$ is finite, then let $m(\alpha) \notin J$. Otherwise, define $m(\alpha)$ arbitrarily.

Let $g: S \rightarrow \omega_{1}$ and $j: S \rightarrow[\omega]^{<\omega}$ be as in Lemma $3\left(\left\{x_{\alpha}: \alpha \in S\right\}\right.$ is reindexed by $\kappa$ in this application of Lemma 3 ).

Let

$$
B=\left\{\alpha \in S: x_{\alpha} \notin \overline{\bigcup\left\{U_{g(\beta)}(\beta): \beta<\alpha\right\}}\right\} .
$$

$\left\{x_{\alpha}: \alpha \in B\right\}$ is separated and so $B$ is nonstationary. Find $\alpha \in S-B$ such that $g\left\lceil\alpha\right.$ and $j \uparrow \alpha=j_{\alpha}$. The conclusion of Lemma $3, j \uparrow \alpha=j_{\alpha}$ and $g \uparrow \alpha=g_{\alpha}$ imply that $\left\{j_{\alpha}(\beta): U_{g(\alpha)}(\alpha) \cap U_{g_{\alpha}(\beta)}(\beta) \neq \emptyset\right.$ and $\left.\beta<\alpha\right\}$ is finite (adding the restriction $\beta<\alpha$ only makes the set smaller). In the definition of $m$, a neighborhood $V$ did exist (for example $U_{g(\alpha)}(\alpha)$ ). $\alpha \in S-B$ implies that $x_{\alpha} \in \overline{U\left\{U_{g(\beta)}(\beta): \beta<\alpha\right\}}$ which implies that there is $\beta_{0}<\alpha$ such that $\left(U_{g(\alpha)}(\alpha) \cap V\right) \cap U_{g\left(\beta_{0}\right)}\left(\beta_{0}\right) \neq \emptyset$. $V \cap U_{g_{\alpha}\left(\beta_{0}\right)}\left(\beta_{0}\right) \neq \emptyset$ implies that $j_{\alpha}\left(\beta_{0}\right) \subset J, m(\alpha) \notin J$ implies $m(\alpha) \notin j_{\alpha}\left(\beta_{0}\right)$. $U_{g(\alpha)}(\alpha) \cap U_{g\left(\beta_{0}\right)}\left(\beta_{0}\right) \neq \emptyset$ implies that $m(\alpha) \in j\left(\beta_{0}\right)$, which is a contradiction.

The proof of Lemma $2(\mathrm{a})$ is more complicated than the proof of Lemma 4 because of the necessity of trapping each neighborhood assignment on its associated stationary set.

PROOF OF LEMMA 2(a). If $\kappa=\omega$, then we use the regularity of $X$. We assume therefore that $\kappa$ is uncountable. We use $V=L$; more specifically Fleissner's $\diamond_{s s}^{\kappa}$ which follows from $V=L$ and which asserts that: If $\left\{A_{f}: f \in{ }^{\kappa} \kappa\right\}$ is a family of stationary sets such that $f \uparrow \alpha=g \uparrow \alpha$ implies $A_{f} \cap(\alpha+1)=A_{g} \cap(\alpha+1)$, then there is a sequence $\left\{f_{\alpha}: \alpha \in \kappa\right\}$ where $f_{\alpha}: \alpha \rightarrow \alpha$ such that, for any $f: \kappa \rightarrow \kappa$, there are stationarily many $\alpha \in A_{f}$ such that $f \uparrow \alpha=f_{\alpha}$. We use the following variation of $\diamond_{s s}^{\kappa}$.

(*) If $\left\{A_{f}: f \in{ }^{\kappa} \kappa\right\}$ is a family of stationary sets such that $f \uparrow \alpha=g \mid \alpha$ implies $A_{f} \cap(\alpha+1)=A_{g} \cap(\alpha+1)$, there there is a sequence $\left\{\left(g_{\alpha}, j_{\alpha}\right): \alpha<\kappa\right\}$ where $g_{\alpha}: \alpha \rightarrow \alpha$ and $j_{\alpha}: \alpha \rightarrow[\omega]^{<\omega}$ such that for any $(g, j)$ where $g: \kappa \rightarrow \kappa$ and $j: \kappa \rightarrow[\omega]^{<\omega}$, there are stationarily many $\alpha \in A_{g}$ such that $g \uparrow \alpha=g_{\alpha}$ and $j \uparrow \alpha=j_{\alpha}$.

The proof that $(*)$ is a variation of $\diamond_{s s}^{\kappa}$ is a standard coding argument; examples of these arguments are found in, for example, [13, p. 32 or 14, p. 85].

We shall code neighborhood assignments to $\left\{x_{\alpha}: \alpha \in \kappa\right\}$ by functions in ${ }^{\kappa} \kappa$. By an argument of Fleissner (Lemma 1 in [1]), if there is an $f: \kappa \rightarrow \kappa$ such that $A_{f}=\left\{\alpha \in \kappa: \overline{\bigcup\left\{U_{f(\beta)}(\beta): \beta<\alpha\right\}} \cap\left\{x_{\beta}: \alpha \leq \beta<\kappa\right\} \neq \emptyset\right\}$ is nonstationary, then $\left\{x_{\alpha}: \alpha<\kappa\right\}$ may be separated by disjoint open sets contrary to assumption. We note that one need only use the collectionwise Hausdorff property for closed discrete sets of cardinality less than $\kappa$ and not normality in this argument. Thus we may assume that each $A_{f}$ is a stationary set. Whether $\alpha \in A_{f}$ depends only on $f \uparrow \alpha$, 
$A_{f} \cap(\alpha+1)$ depends only on $f \uparrow \alpha$, and thus $\left\{A_{f}: f \in{ }^{\kappa} \kappa\right\}$ is as in the hypothesis of $(*)$. Let $\left\{\left(g_{\alpha}, j_{\alpha}\right): \alpha<\kappa\right\}$ be as in the conclusion of $(*)$.

We will define a partial function $m: \kappa \rightarrow \omega$ inductively.

The inductive definition of the partial function $m: \kappa \rightarrow \omega$ will be somewhat complicated. We will want to keep track of the stages of the induction at which "something happened", so we will also define an auxiliary function $e: \kappa \rightarrow 2$. $e(\alpha)=$ 1 will tell us that "something happened" at stage $\alpha$.

Suppose that $e \uparrow \alpha$ has been defined but $e(\alpha)$ has not yet been defined. If

$$
\overline{\bigcup\left\{U_{g_{\alpha}(\beta)}(\beta): \beta<\alpha\right\}} \cap\left\{x_{\beta}: \alpha \leq \beta<\kappa\right\}=\emptyset,
$$

then let $e(\alpha)=1$ and do nothing else. Otherwise, choose $\alpha^{\prime} \geq \alpha$ such that $x_{\alpha^{\prime}} \in$ $\bar{\bigcup}\left\{U_{g_{\alpha}(\beta)}(\beta): \beta<\alpha\right\}$. Let $e(\alpha)=1$ and let $e(\beta)=0$ for each $\alpha<\beta \leq \alpha^{\prime}$.

If there is a neighborhood $V$ of $x_{\alpha^{\prime}}$ such that $J=\bigcup\left\{j_{\alpha}(\beta): U_{g_{\alpha}(\beta)}(\beta) \cap V \neq\right.$ $\emptyset$ and $\beta<\alpha\}$ is finite, then let $m\left(\alpha^{\prime}\right)$ be a natural number which is not an element of $J$. Otherwise, do nothing else.

Now there is some $\beta>\alpha$ such that $e \uparrow \beta$ has been defined but $e(\beta)$ has not yet been defined.

Note that $e^{-1}(\{1\})$ is a closed unbounded set. That is, something happened on a closed unbounded set.

Let $g: \kappa \rightarrow \lambda$ and $j: \kappa \rightarrow[\omega]^{<\omega}$ be as in Lemma 3. By $(*)$, there are stationarily many $\alpha \in A_{g}$ such that $g \uparrow \alpha=g_{\alpha}$ and $j \uparrow \alpha=j_{\alpha}$. Recall that $e^{-1}(\{1\})$ is a closed unbounded set and find an $\alpha \in A_{g}$ such that $e(\alpha)=1, g\left\lceil\alpha=g_{\alpha}\right.$ and $j \uparrow \alpha=j_{\alpha}$.

First, note that since $e(\alpha)=1, \alpha$ "came up" in the inductive definition of $m$. That is, as we defined $m$ (and $e$ ), there was a point at which $e \uparrow \alpha$ had been defined and $e(\alpha)$ had not yet been defined.

Let us examine the reasoning which must have taken place at that time. Since $\alpha \in A_{g}, \overline{\bigcup\left\{U_{g(\beta)}(\beta): \beta<\alpha\right\}} \cap\left\{x_{\beta}: \alpha \leq \beta<\kappa\right\} \neq \emptyset$ but since $g \mid \alpha=g_{\alpha}$, $\bigcup\left\{U_{g_{\alpha}(\beta)}(\beta): \beta<\alpha\right\} \cap\left\{x_{\beta}: \alpha \leq \beta<\kappa\right\} \neq \emptyset$. This means we chose $\alpha^{\prime} \geq \alpha$ such that $x_{\alpha^{\prime}} \in \bar{\bigcup}\left\{U_{g_{\alpha}(\beta)}(\beta): \beta<\alpha\right\}$.

By the conclusion of Lemma $3,\left\{j(\beta): U_{g\left(\alpha^{\prime}\right)}\left(\alpha^{\prime}\right) \cap U_{g(\beta)}(\beta) \neq \emptyset\right\}$ is finite and so there exists a neighborhood $V$ of $x_{\alpha^{\prime}}$ such that

$$
\left\{j(\beta): \beta<\alpha \text { and } U_{g(\beta)}(\beta) \cap V \neq \emptyset\right\} \quad \text { is finite }
$$

( $\beta<\alpha$ only makes that finite set smaller). So, knowing that this set is finite, let us continue our examination of the reasoning which took place at that time when $e$ 「 $\alpha$ had been defined but $e(\alpha)$ had not yet been defined. By the above, since $j \uparrow \alpha=j_{\alpha}$ and $g \uparrow \alpha=g_{\alpha}, \bigcup\left\{j_{\alpha}(\beta): \beta<\alpha\right.$ and $\left.U_{g_{\alpha}(\beta)}(\beta) \cap V \neq \emptyset\right\}$ is finite and so $m\left(\alpha^{\prime}\right)$ was defined and

$$
m\left(\alpha^{\prime}\right) \notin J .
$$

Note, however, that $\alpha^{\prime}$ was chosen such that $x_{\alpha^{\prime}} \in \overline{\bigcup\left\{U_{g_{\alpha}(\beta)}(\beta): \beta<\alpha\right\}} . V$ and $U_{g\left(\alpha^{\prime}\right)}\left(\alpha^{\prime}\right)$ are both neighborhoods of $x_{\alpha^{\prime}}$ and so

$$
\left(V \cap U_{g\left(\alpha^{\prime}\right)}\left(\alpha^{\prime}\right)\right) \cap \bigcup\left\{U_{g_{\alpha}(\beta)}(\beta): \beta<\alpha\right\} \neq \emptyset,
$$

so that

$$
V \cap U_{g\left(\alpha^{\prime}\right)}\left(\alpha^{\prime}\right) \cap U_{g_{\alpha}\left(\beta_{0}\right)}\left(\beta_{0}\right) \neq \emptyset \text { for some } \beta_{0}<\alpha
$$


In particular, $U_{g\left(\alpha^{\prime}\right)}\left(\alpha^{\prime}\right) \cap U_{g_{\alpha}\left(\beta_{0}\right)}\left(\beta_{0}\right) \neq \emptyset$ and so by our definition of $j\left(\beta_{0}\right)$,

$$
m\left(\alpha^{\prime}\right) \in j\left(\beta_{0}\right) \text {. }
$$

Also, in particular from (2), $V \cap U_{g_{\alpha}\left(\beta_{0}\right)}\left(\beta_{0}\right) \neq \emptyset$ so by our definition of $J$ (since $\left.\beta_{0}<\alpha\right), j_{\alpha}\left(\beta_{0}\right) \subset J$. Since $j_{\alpha}=j \uparrow \alpha, j\left(\beta_{0}\right) \subset J$ and so by $(3) m\left(\alpha^{\prime}\right) \in J$, which contradicts (1) and ends the proof.

PROOF OF LEMMA 2(b). We assume that $\left\{\kappa_{\beta}: \beta<\operatorname{cf}(\kappa)\right\}$ is a closed unbounded set in $\kappa$ of cardinals, not less that $\lambda$, enumerated in increasing order. We assume, without loss of generality, that $\operatorname{cf}(\kappa) \leq \lambda$. We use only the GCH in the proof of Lemma 2(b). This makes the proof more complicated but there do not seem to be any useful enumeration principles for singular cardinals which are any stronger than the GCH.

The GCH allows the inductive definition of a counterexample to countable paracompactness but by witnessing the failure of each neighborhood assignment to both

(a) separate "most" of the discrete family of points, and

(b) witness the way in which the space is countably paracompact with respect to the partition,

the best that can be obtained from the method of proof of Lemma 2(a) is the following:

LEMMA $5(\mathrm{GCH})$. There is a neighborhood assignment $f: \kappa \rightarrow \lambda$ such that for each $\lambda \leq \alpha<\kappa, \bar{U}\left\{U_{f(\beta)}(\beta): \beta<\alpha\right\} \cap\left\{x_{\beta}: \beta<\kappa\right\}$ has cardinality at most $|\alpha|$.

PROOF. We use GCH, more specifically, the following principle: $(\Omega)$ There is a sequence $\left\{\left(g_{\alpha}, j_{\alpha}\right): \lambda \leq \alpha<\kappa\right\}$ where $g_{\alpha}$ is a partial function from $\kappa$ into $\lambda$ and $j_{\alpha}$ is a partial function from $\kappa$ into $[\omega]^{<\omega}$ such that for any $(g, j)$ where $g: \kappa \rightarrow \lambda$ and $j: \kappa \rightarrow[\omega]^{<\omega}$ and any $\beta \in[\lambda, \kappa)$, there is an $\alpha$ such that $g \uparrow \beta=g_{\alpha}, j \uparrow \beta=j_{\alpha}$ and $|\beta|=|\alpha|$.

To see that $(\Omega)$ follows from GCH whenever $\kappa$ is a limit cardinal, let $\left\{\left(g_{\alpha}, j_{\alpha}\right): \mu \leq\right.$ $\left.\alpha<\mu^{+}\right\}$list $\left\{(g, j): g: \eta \rightarrow \lambda, j: \eta \rightarrow[\omega]^{<\omega}\right.$ for some $\left.\eta \in\left[\mu, \mu^{+}\right)\right\}$whenever $\mu \in$ $[\lambda, \kappa)$ is a cardinal.

Modify the proof of Lemma 4 by letting $S=\kappa, g: \kappa \rightarrow \lambda$ and $m: \kappa \rightarrow \omega$ be a partial function defined in an induction of length $\kappa$. At stage $\alpha \in \kappa$, if there is $\gamma$ not yet in the domain of $m$ such that $x_{\gamma} \in \overline{\bigcup\left\{U_{g_{\alpha}(\beta)}(\beta): \beta \in \operatorname{dom}\left(g_{\alpha}\right)\right\}}$ and if $J$ (as in Lemma 4 except $\beta$ ranges over $\left.\operatorname{dom}\left(j_{\alpha}\right)\right)$ is finite, then let $m(\gamma) \notin J$. If there is an $\alpha \in[\lambda, \kappa)$ such that $\overline{\bigcup\left\{U_{g(\beta)}(\beta): \beta<\alpha\right\}} \cap\left\{x_{\beta}: \beta<\kappa\right\}|>| \alpha \mid$, then, by $(\Omega)$, there is a $\beta$ such that $g\left\lceil\alpha=g_{\beta}, j\left\lceil\alpha=j_{\beta}\right.\right.$ and $|\beta|=|\alpha|$.

Revisiting the reasoning which took place at stage $\beta$ of the inductive definition of $m$ shows that there is $\gamma \in \kappa$ such that $m(\gamma)$ lies both in and out of $J$ as before.

We can shorten the exposition of the proof of Lemma 2(b) by making an observation: The hypothesis of normality for Lemma 1 may be weakened to normality for closed discrete sets, and so Lemma 1 shows that to prove Lemma 2(b), it suffices to show the following:

LEMMA $6 . X$ is normal with respect to closed discrete sets of cardinality at most $\kappa$.

It is somewhat simpler to show that a discrete family of points is normalized than separated. For example, Lemma 5 yields the following weak form of Lemma 6 . 
LEMMA 7. If $A, B$ are disjoint subsets of $\left\{x_{\alpha}: \alpha \in \kappa\right\}$ such that $|A|<|B|=\kappa$, then there are disjoint open sets $U, V$ such that $U \supset A$ and $V \supset B$.

PROOF. Reorder $\left\{x_{\alpha}: \alpha \in \kappa\right\}$ so that $A$ has indices bounded by $\mu$ where $\lambda \leq$ $\mu<\kappa$. Apply Lemma 5 to $\left\{x_{\alpha}: \alpha<\kappa\right\}$ to get a neighborhood assignment $f: \kappa \rightarrow \lambda$ such that $\bigcup\left\{U_{f(\beta)}(\beta): \beta<\mu\right\} \cap\left\{x_{\alpha}: \alpha<\kappa\right\}$ has cardinality at most $\mu$.

Assume, without loss of generality, that $x_{\alpha} \notin \overline{\bigcup\left\{U_{f(\beta)}(\beta): \beta \in \mu\right\}}$ implies

$$
U_{f(\alpha)}(\alpha) \cap U_{f(\beta)}(\beta)=\emptyset \quad(\beta \in \mu)
$$

and that $x_{\alpha} \in \overline{\bigcup\left\{U_{f(\beta)}(\beta): \beta \in \mu\right\}}$ implies

$$
U_{f(\alpha)}(\alpha) \cap U_{f(\beta)}(\beta) \quad(\beta \in \mu: \beta \neq \alpha)
$$

(using the collectionwise Hausdorff property for closed discrete sets of cardinality $\mu)$.

ProOF OF LEMMA 6. We let $\left\{x_{\alpha}: \alpha<\kappa\right\}$ and $\left\{y_{\alpha}: \alpha<\kappa\right\}$ be disjoint closed discrete sets. We wish to separate $\left\{x_{\alpha}: \alpha<\kappa\right\}$ and $\left\{y_{\alpha}: \alpha<\kappa\right\}$.

Let us use what we can of Lemma 5 in the simplified context of Lemma 6:

LEMMA 8. There are neighborhood assignments $f: \kappa \rightarrow \lambda$ and $g: \kappa \rightarrow \lambda$ and $a$ subset $E$ of $\kappa$ such that, for any $\alpha, \beta \in \kappa-E, U_{f(\alpha)}\left(x_{\alpha}\right) \cap U_{g(\beta)}\left(y_{\beta}\right)=\emptyset$ and such that for any $\beta<\operatorname{cf}(\kappa),\left|E \cap\left[\kappa_{\beta}, \kappa_{\beta+1}\right)\right| \leq \kappa_{\beta}$.

Lemma 8 states that we can separate "most" of $\left\{x_{\alpha}: \alpha<\kappa\right\}$ from "most" of $\left\{y_{\alpha}: \alpha<\kappa\right\}$.

PROOF. List $\left\{x_{\alpha}: \alpha \in \kappa\right\} \cup\left\{y_{\alpha}: \alpha \in \kappa\right\}$ by $\left\{a_{\alpha}: \alpha \in \kappa\right\}$ so that $x_{\alpha}$ and $y_{\alpha}$ appear consecutively. Let $f^{*}$ be a neighborhood assignment as in Lemma 5 . Assume, without loss of generality, that $f^{*}$ separates $\left\{x_{\alpha}: \kappa_{\beta} \leq \alpha<\kappa_{\beta+1}\right\}$ and $\left\{y_{\alpha}: \kappa_{\beta} \leq\right.$ $\alpha<\kappa_{\beta+1}$ \} whenever $\beta \in \operatorname{cf}(\kappa)$ (applying the collectionwise Hausdorff property for closed discrete sets of cardinality less than $\kappa)$ and that $a_{\alpha} \notin \overline{\bigcup\left\{U_{f^{*}(\beta)}(\beta): \beta \in \alpha\right\}}$ implies $U_{f^{*}(\alpha)}(\alpha) \cap U_{f^{*}(\beta)}(\beta)=\emptyset$ whenever $\beta \in \alpha$. $f^{*}$ induces $f$ and $g$ by returning to the original indexing.

Let

$$
\begin{aligned}
E= & \left\{\alpha: y_{\alpha} \in \overline{\bigcup\left\{U_{f(\eta)}\left(x_{\eta}\right): \eta \in \alpha\right\}}\right\} \\
& \cup\left\{\alpha: x_{\alpha} \in \overline{\bigcup\left\{U_{g(\eta)}\left(y_{\eta}\right): \eta \in \alpha\right\}}\right\} .
\end{aligned}
$$

By Lemma 5 , for each $\beta \in \operatorname{cf}(\kappa),\left|E \cap\left[\kappa_{\beta}, \kappa_{\beta+1}\right)\right| \leq \kappa_{\beta}$.

Let us state the crucial property of "most" which enables the proof of Lemma 6 to work:

LEMMA 9. Let $E$ be a subset of $\kappa$ such that $|E|=\kappa$ and such that for any ordinal $\beta<\operatorname{cf}(\kappa),\left|E \cap\left[\kappa_{\beta}, \kappa_{\beta+1}\right)\right| \leq \kappa_{\beta}$. There is an ordering $\left\{a_{\alpha}: \alpha<\kappa\right\}$ of $E$ such that, for all $\beta \in E$, if $\beta \geq \kappa_{0}$, then $\beta=a_{\alpha}$ for some $\alpha<\beta$.

Lemma 9 states that the complement of "most" of $\kappa$ may be reindexed by an eventually regressive function.

PROOF. For each $\beta<\operatorname{cf}(\kappa)$, let $\left[\kappa_{\beta}, \kappa_{\beta+1}\right)$ be partitioned as $\left\{E_{\alpha}^{\beta}: \alpha<\operatorname{cf}(\kappa)\right\}$ where $\left|E_{\alpha}^{\beta}\right|=\kappa_{\beta+1}$ for each $\alpha<\operatorname{cf}(\kappa)$. Note that for each $1 \leq \alpha<\operatorname{cf}(\kappa)$,

$$
\left|\bigcup\left\{E_{\alpha}^{\beta}: \beta<\alpha\right\}\right|=\sum\left\{\kappa_{\beta+1}: \beta<\alpha\right\}=\kappa_{\alpha} .
$$


Further, note that $\left\{\bigcup\left\{E_{\alpha}^{\beta}: \beta<\alpha\right\}: \alpha<\operatorname{cf}(\kappa)\right\}$ is a partition of $\left[\kappa_{0}, \kappa\right)$. Let $\kappa_{0}$ be partitioned into two sets $C_{1}, C_{2}$ such that $\left|C_{1}\right|=\left|C_{2}\right|=\kappa_{0}$. For each $\beta<\operatorname{cf}(\kappa)$, $\left|E \cap\left[\kappa_{\beta}, \kappa_{\beta+1}\right)\right| \leq \kappa_{\beta}$. So map $E \cap\left[\kappa_{\beta}, \kappa_{\beta+1}\right)$ injectively into $\bigcup\left\{E_{\beta}^{\nu}: \nu<\beta\right\}$ for each $\beta \geq 1$. Map $E \cap\left[\kappa_{0}, \kappa_{1}\right)$ injectively into $C_{1}$ and map $E \cap \kappa_{0}$ injectively into $C_{2}$. The union of these injections is an injection since their ranges are disjoint.

If $\kappa_{1} \leq \lambda \in E$, then $\lambda$ lies in $E \cap\left[\kappa_{\beta}, \kappa_{\beta+1}\right)$ for some $1 \leq \beta<\operatorname{cf}(\kappa) . \lambda$ is then mapped into $\left\{E_{\beta}^{\nu}: \nu<\beta\right\}$ and thus into some $E_{\beta}^{\nu} \subset\left[\kappa_{\nu}, \kappa_{\nu+1}\right)$. The image of $\lambda$ is less than $\kappa_{\nu+1} \leq \kappa_{\beta} \leq \lambda$ as required. If $\kappa_{0} \leq \lambda<\kappa_{1}$, then $\lambda$ is mapped into $C_{1}$ and so the image of $\lambda$ is less than $\kappa_{0}$ as required and Lemma 9 is proved.

PROOF OF LEMMA 6 (CONTINUED). In this proof, we apply Lemma 8 in an iteration along an $\omega$-tree. At each node $s$, there is a subset $A_{s}$ of $\left\{x_{\alpha}: \alpha<\kappa\right\}$ and a subset $B_{s}$ of $\left\{y_{\alpha}: \alpha<\kappa\right\}$ which we wish to separate. We apply Lemma 8 to separate most of $A_{s}$ from most of $B_{s}$. This leaves a task for each of two successor nodes: to separate $A$ from all but most of $B$ and to separate all but most of $A$ from $B$. Each successor of a node $s$ reindexes either $A_{s}$ or $B_{s}$ regressively so that each ordered pair $\left(x_{\alpha}, y_{\alpha}\right)$ "occurs" in a finite subtree.

There are two complications.

First, the tree is not defined by level but, instead, in such a way that the subsets of $\left\{x_{\alpha}: \alpha<\kappa\right\}$ are uniformly indexed. Second, it is necessary to apply the countable paracompactness of $X$ with respect to the partition of $\left\{x_{\alpha}: \alpha<\kappa\right\}$ induced by the uniform reindexing and the partition of $\left\{y_{\alpha}: \alpha<\kappa\right\}$ induced by the association of a finite subtree to each $y_{\alpha}$.

Let $\Xi$ be the set of finite strings of zeros and ones. We shall define $\left\{\left(A_{n}, B_{s}\right): s \in\right.$ $\Xi ; n \in \omega\}$ where each $A_{n}$ is an indexing of a subset of $\left\{x_{\alpha}: \alpha \in \kappa\right\}$ and each $B_{s}$ is an indexing of a subset of $\left\{y_{\alpha}: \alpha \in \kappa\right\}$ by induction on $n$ and the number of zeros in $s$. The 0 th stage consists of defining $A_{0}=\left\{x_{\alpha}: \alpha \in \kappa\right\}$ and $B_{\emptyset}=\left\{y_{\alpha}: \alpha \in \kappa\right\}$. The $n$th stage of the induction (for $n \geq 1$ ) takes place in two parts: In the first part, we define $\left(A_{n}, B_{s}\right)$ for strings $s$ which have precisely $n-1$ many zeros and which end in a one. In the second part, we define $\left(A_{n}, B_{s}\right)$ for strings $s$ which have precisely $n$ many zeros and which end in a zero.

Let us describe the first part: Let $t$ be a fixed string occurring at stage $n-1$ which ends in a zero. We define $B_{s}$, where $s=t \widehat{u}$ and $u$ is a finite string of ones, by a subinduction on the length of $u$.

Apply Lemma 8 to $\left(A_{n-1}, B_{t^{\wedge} u}\right)$, where $u$ is a (possibly empty) string of ones, to get neighborhood assignments $f_{t^{\wedge} u}^{*}, g_{t^{\wedge} u}^{*}$ and a subset $E$ of $\kappa$. Let $B_{t^{\wedge} u^{\wedge} 1}$ be the indexing with domain $B_{t^{\wedge} u}^{-1}(E)$ given by an application of Lemma 9 to $B_{t^{\wedge} u}\left(B_{t^{\wedge} u}^{-1}(E)\right)$.

Let us describe the second part: We define $A_{n}$. Apply Lemma 8 to each $\left(A_{t}, B_{t}\right)$ to which Lemma 8 has not yet been applied to get neighborhood assignments $f_{t}^{*}, g_{t}^{*}$ and a subset $E_{t}$ of $\kappa$. Let $E$ be the union of the $E_{t}$ (the union is taken over all $t$ being considered in this paragraph). $A_{n}$ is the indexing with domain $A_{n-1}^{-1}(E)$ given by an application of Lemma 9 to $A_{n-1}\left(A_{n-1}^{-1}(E)\right)$.

Let $M=\left\{\delta\right.$ : there is an $s \in \Xi$ and an $\alpha<\lambda$ such that either $x_{\delta}$ is the $\alpha$ th element of $A_{s}$ under its associated ordering or $y_{\delta}$ is the $\alpha$ th element of $B_{s}$ under its associated ordering $\}$. We compute the cardinality of $M$ to be $\lambda$.

Note than we can define $f$ and $g$ such that whenever

(1) $\delta \in M$ and $\gamma \notin M, U_{f(\delta)}\left(x_{\delta}\right) \cap U_{g(\gamma)}\left(y_{\gamma}\right)=U_{f(\gamma)}\left(x_{\gamma}\right) \cap U_{g(\delta)}\left(y_{\delta}\right)=\emptyset$ by Lemma 7 , and such that 
(2) whenever $\delta, \gamma \in M, U_{f(\delta)}\left(x_{\delta}\right) \cap U_{g(\gamma)}\left(y_{\gamma}\right)=\emptyset$

by the collectionwise Hausdorff property for closed discrete sets of cardinality less than $\kappa$.

We note that, whenever $\delta, \gamma \notin M$, there is a string $s$ with $n$ zeros such that $x_{\delta} \in A_{n}-A_{n+1}$ and $y_{\gamma} \in B_{s}-B_{s \wedge 1}$. Otherwise, for each string $s$ with $n$ zeros such that $x_{\delta} \in A_{n}$ and $y_{\gamma} \in B_{s}$, either $x_{\delta} \in A_{n+1}$ or $y_{\gamma} \in B_{s^{\wedge} 1}$. Since $x_{\delta} \in A_{0}$ and $y_{\gamma} \in B_{\emptyset}$, by induction there is a sequence of strings $\left\{s_{i}: i \geq 0\right\}$ such that the length of $s_{i}$ is $i$, the $s_{i}$ are linearly ordered by inclusion and $x_{\delta} \in A_{n_{i}}$ and $y_{\gamma} \in B_{s_{i}}$ (where $n_{i}$ is the number of zeros in $s_{i}$ ). We applied Lemma 9 in the indexing of $A_{n}$ so that the index of $x_{\delta}$ in $A_{n+1}$ is less than the index of $x_{\delta}$ in $A_{n}(\delta \notin M$ implies that the index of $x_{\delta}$ is not less than $\lambda$ ). There is no infinite decreasing sequence of ordinals implies that the number of zeros in the $s_{i}$ is bounded.

We applied Lemma 9 in the indexing of $B_{s_{i}}$ so the index of $y_{\gamma}$ in $B_{s_{i+1}}$ is less than the index of $y_{\gamma}$ in $B_{s_{i}}$ whenever $s_{i+1}=s_{i} \widehat{ } 1(\gamma \notin M$ implies that the index of $y_{\gamma}$ in $B_{s_{i}}$ is not less than $\lambda$ ). There is no infinite decreasing sequence of ordinals implies that $s_{i+1}=s_{i} \widehat{ }{ }^{\widehat{1}}$ for at most finitely many $i$. This is a contradiction with the bounded number of zeros in the $s_{i}$.

$\left\{A_{i}-A_{i+1}: i \geq 0\right\}$ is a discrete family of closed subsets of $\left\{x_{\alpha}: \alpha \in \kappa\right\}$. Let $\left\{U_{n}: n \geq 0\right\}$ be a locally finite family of open sets such that $U_{n} \cap\left\{x_{\alpha}: \alpha \in \kappa\right\}=$ $A_{i}-A_{i+1}$. We can define $f$ and $g$ such that

(3) $f \uparrow(\kappa-M)$ refines $\left\{U_{n}: n \geq 0\right\}$ and $g$ witnesses the local finiteness of $\left\{U_{n}: n \geq 0\right\}$.

For each $\alpha \in \kappa-M$, letting $n(\alpha)=\max \left\{n: U_{g(\alpha)}\left(y_{\alpha}\right) \cap U_{n} \neq \emptyset\right\}$, consider the set of strings $s$ such that $s$ has at most $n(\alpha)$ many zeros and $y_{\alpha} \in B_{s}$. This is a subtree $T_{\alpha}$ of . Any infinite branch in $T_{\alpha}$ necessitates an infinite decreasing sequence of ordinals, so there are no infinite branches in $T_{\alpha}$. By Konig's Lemma, $T_{\alpha}$ is a finite tree. We can define $g$ such that

(4) whenever $\gamma \in \kappa-M, s \in T_{\gamma}$, the $\beta$ th element of $B_{s}$ is $y_{\gamma}$ and $y_{\gamma} \in B_{s}-B_{s^{\wedge} 1}$, $U_{g(\gamma)}\left(y_{\gamma}\right) \subset U_{g_{s}^{*}(\beta)}\left(y_{\gamma}\right)$.

For each $\alpha \in \kappa-M$, we have associated a finite tree $T_{\alpha}$. Let the finite trees be enumerated as $\left\{T_{n}: n \geq 0\right\}$. Let $\left\{y_{\alpha}: \alpha \in(\kappa-M)\right\}$ be partitioned as follows: $\left\{\left\{y_{\alpha}: \alpha \in(\kappa-M)\right.\right.$ and $\left.\left.T_{\alpha}=T_{n}\right\}: n \geq 0\right\}$. Applying countable paracompactness, let $\left\{V_{n}: n \geq 0\right\}$ be a locally finite family of open sets such that $V_{n} \cap\left\{y_{\alpha}: \alpha<\kappa\right\}=$ $\left\{y_{\alpha}: \alpha \in \kappa-M\right.$ and $\left.T_{\alpha}=T_{n}\right\}$.

We can define $f$ and $g$ such that

(5) $g$ refines $\left\{V_{n}: n \geq 0\right\}$ on $\left\{y_{\alpha}: \alpha \in \kappa-M\right\}$ and $f$ witnesses the local finiteness of $\left\{V_{n}: n \geq 0\right\}$.

For each $\delta \in \kappa-M$, let $N(\delta)=\left\{n \geq 0: U_{f(\delta)}\left(x_{\delta}\right) \cap V_{n} \neq \emptyset\right\}$. Let $T(\delta)=$ $\bigcup\left\{T_{n}: n \in N(\delta)\right\}$.

We can define $f$ such that

(6) whenever $\delta \in \kappa-M, s \in T(\delta)$ and the $\beta$ th element of $A_{s}$ is $x_{\delta}, U_{f(\delta)}\left(x_{\delta}\right) \subset$ $U_{f_{s}^{*}(\beta)}\left(x_{\delta}\right)$. Since $T(\delta)$ is finite, this is possible.

We define $f$ and $g$ to be such that (1)-(6) hold. Suppose $U_{f(\delta)}\left(x_{\delta}\right) \cap U_{g(\gamma)}\left(y_{\gamma}\right) \neq$ $\emptyset$. By (1) and (2), we may assume $\delta, \gamma \notin M$.

There is a string $s$ with $n$ zeros such that $x_{\delta} \in A_{n}-A_{n+1}$ and $y_{\gamma} \in B_{s}-B_{s^{\wedge} 1}$. By (3) and the definition of $n(\gamma), U_{g(\gamma)}\left(y_{\gamma}\right) \cap U_{n}=\emptyset$ for any $n>n(\gamma)$. That is, $U_{g(\gamma)}\left(y_{\gamma}\right) \cap U_{f(\delta)}\left(x_{\delta}\right)=\emptyset$ for any $x_{\delta} \in A_{n}-A_{n+1} ; n>n(\gamma)$. In other words, $s$ has 
no more than $n(\gamma)$ many zeros. However, $y_{\gamma} \in B_{s}$, thus $s \in T_{\gamma}$. By (4), when the $\beta$ th element of $B_{s}$ is $y_{\gamma}, U_{g(\gamma)}\left(y_{\gamma}\right) \subset U_{g_{s}^{*}(\beta)}\left(y_{\gamma}\right)$. Suppose $T_{\gamma}=T_{k}$ and $y_{\gamma} \in V_{k}$. If $k \notin N(\delta)$, then $U_{f(\delta)}\left(x_{\delta}\right) \cap V_{k}=\emptyset$ and $U_{f(\delta)}\left(x_{\delta}\right) \cap U_{g(\gamma)}\left(y_{\gamma}\right)=\emptyset$ by $(5)$.

So we may assume $k \in N(\delta)$. Thus $T(\delta) \supset T_{k}=T_{\gamma}$ which contains $s$.

By (6), if the $\beta$ th element of $A_{s}$ is $x_{\delta}, U_{f(\delta)}\left(x_{\delta}\right) \subset U_{f_{s}^{*}(\beta)}\left(x_{\delta}\right)$.

By the definition of $f_{s}^{*}$ and $g_{s}^{*}, U_{g_{s}^{*}(\beta)}\left(y_{\gamma}\right) \cap U_{f_{s}^{*}(\alpha)}\left(x_{\delta}\right)=\emptyset$ and so $U_{g(\gamma)}\left(y_{\gamma}\right) \cap$ $U_{g(\delta)}\left(x_{\delta}\right)=\emptyset$, which is a contradiction.

In $[\mathbf{1 5}]$, we used Lemma 1 of this section to show that, under $V=L$, locally compact normal spaces are collectionwise Hausdorff and that, under $V=L$, locally compact, metacompact normal spaces are paracompact. A natural conjecture is that Lemma 2 of this section can be used to show that, under $V=L$, locally compact countably paracompact spaces are collectionwise Hausdorff and that, under $V=L$, locally compact metacompact countably paracompact spaces are paracompact. We have been unable, however, to prove this conjecture. Balogh [17] has shown that, under $V=L$, locally compact metacompact countably paracompact spaces are paracompact.

Daniels [18] has shown that, under $V=L$, locally compact countably paracompact spaces of character less than $\aleph_{\omega}$ are collectionwise Hausdorff. A question remains:

Question 1 . Is there, in $\mathrm{ZFC}$, a locally compact countably paracompact space which is not collectionwise Hausdorff?

Kunen and Nyikos [20] showed that the Product Measure Extension Axiom (which can be shown consistent, using a strongly compact cardinal) implies that normal first countable spaces are collectionwise normal and so that normal Moore spaces are metrizable.

Burke [19] has shown that this axiom also implies that countably paracompact first countable spaces are collectionwise normal for subparacompact sets and so that countably paracompact Moore spaces are metrizable.

\section{Separable countably paracompact spaces.}

THEOREM 2. There is a countably paracompact separable space with an uncountable closed discrete set if and only if there is a dominating family in ${ }^{\omega_{1}} \omega$ of cardinality of the continuum.

To motivate this result, we state a result of Steprans, Jech and Prikry: $\left(2^{\aleph_{0}}<2^{\aleph_{1}}\right.$ and $2^{\aleph_{0}}$ is a regular cardinal and there is no measurable cardinal in an inner model) implies that there is no dominating family in ${ }^{\omega_{1}} \omega$ of cardinality $2^{\aleph_{0}}$. This implies that under $\left(2^{\aleph_{0}}<2^{\aleph_{1}}\right.$ and $2^{\aleph_{0}}$ is a regular cardinal and there is no measurable cardinal in an inner model) countably paracompact separable spaces are collectionwise Hausdorff.

PROOF OF THEOREM 2. First, we assume that there is a countably paracompact, separable space with an uncountable closed discrete set and show that there is a dominating family in ${ }^{\omega_{1}} \omega$ of cardinality of the continuum.

Let $X$ be a countably paracompact space where $\omega_{1}-\omega$ is a closed discrete set and $\omega$ is a dense set. Let $\left\{\left\{P_{n}^{\alpha}: n \in \omega\right\}: \alpha<\kappa\right\}$, where $\kappa \leq 2^{\aleph_{0}}$, enumerate the locally finite sequences of subsets of $\omega$. For each $\alpha<\kappa$, let $f_{\alpha}:\left(\omega_{1}-\omega\right) \rightarrow \omega$ be defined by $f_{\alpha}(\beta)=\max \left\{n: \beta \in \bar{P}_{n}^{\alpha}\right\}$. Claim that $\left\{f_{\alpha}: \alpha<\kappa\right\}$ is a dominating family in $\left(\omega_{1}-\omega\right) \omega$. Let $g: \omega_{1}-\omega \rightarrow \omega$. $\left\{g^{-1}(n): n \in \omega\right\}$ is a partition of $\omega_{1}-\omega$, and so there 
is a locally finite family $\left\{U_{n}: n \in \omega\right\}$ of open sets such that $U_{n} \cap\left(\omega_{1}-\omega\right)=g^{-1}(n)$. $\left\{U_{n} \cap \omega: n \in \omega\right\}$ is a locally finite sequence of subsets of $\omega$ and so there is an $\alpha<\kappa$ such that, for each $n \in \omega, U_{n} \cap \omega=P_{n}^{\alpha} . g^{-1}(n)$ is contained in $U_{n}$ which is contained in $\overline{U_{n}}=\overline{U_{n} \cap \omega}=\overline{P_{n}^{\alpha}}$. If $g(\beta)=n$, then $\beta \in g^{-1}(n)$ and so $\beta \in \overline{P_{n}^{\alpha}}$ and $f_{\alpha}(\beta) \geq n$. We have shown that there is an $\alpha<\kappa$ such that, for each $\beta \in \omega$, $f_{\alpha}(\beta) \geq g(\beta)$ as required.

Second, we assume that there is a dominating family in ${ }^{\omega_{1}} \omega$ of cardinality of the continuum and show that there is a countably paracompact separable space with an uncountable closed discrete set. Let $\left\{f_{\beta}: \beta<2^{\omega}\right\}$ be a dominating family in ${ }^{\omega_{1}} \omega$. Assume, without loss of generality, that, for each $\alpha \in \omega_{1}$, there is a $\beta \in 2^{\omega}$ such that $f_{\beta}^{-1}(\{0\})=\{\alpha\}$ (this is a technical point which is needed so that the space constructed is regular). Let $X$ be the set of functions with domain $2^{\omega}$ and range $\omega$. Let $X$ have the product topology. Let $\pi: \omega_{1} \rightarrow X$ be defined by $\pi(\alpha)(\beta)=f_{\beta}(\alpha)$. Let $D=\left\{d_{i}: i \in \omega\right\}$ be a countable dense subset of $X$. $D$ exists since the product of continuum-many separable spaces is separable. Let $Y$ be the union of the range of $\pi$ and $D$. Let $Y$ have the topology obtained by refining the subspace topology by isolating each element of $D$. Claim that $Y$ is a countably paracompact space such that $D$ is dense in $Y$ and the range of $\pi$ is an uncountable closed discrete set. We show that the range of $\pi$ is an uncountable closed discrete set. Let $\alpha \in \omega_{1}$. By the additional assumption on the dominating family, there is a $\beta \in 2^{\omega}$ such that $f_{\beta}(\alpha)=0$ and such that for any $\alpha \neq \alpha^{\prime}$, $f_{\beta}\left(\alpha^{\prime}\right)=1$ and so $\pi$ is an injection and the range of $\pi$ is an uncountable set. $U=Y \cap\{f: f(\beta)=0\}$ is a basic open neighborhood of $Y$ and $\operatorname{rng}(\pi) \cap U=$ $\{\pi(\alpha)\}$. Thus, since $D$ is a set of isolated points, each point in $Y$ has a neighborhood which intersects $\operatorname{rng}(\pi)$ in at most one point and $\operatorname{rng}(\pi)$ is an uncountable closed discrete set as required. $Y$ is separable since any basic open set of $Y$ is either a subset of $D$ of cardinality 1 (and intersects $D$ ) or is the intersection of a basic open set of $X$ with $Y$ (and intersects $D$ ). $Y$ is completely regular since $X$ is a 0-dimensional Hausdorff space (the 0-dimensional Hausdorff property is productive and preserved by isolating points). We show that $Y$ is countably paracompact. Let $\left\{U_{n}: n \in \omega\right\}$ be an open cover of $Y$. Let $\left\{W_{n}: n \in \omega\right\}$ be a partition of $\omega_{1}$ such that $\pi\left(W_{n}\right)$ is contained in $U_{n} \cap \operatorname{rng}(\pi)$. Let $g: \omega_{1} \rightarrow \omega$ be defined by $g(\beta)=n$ if $\beta \in W_{n}$. There is an $\alpha \in 2^{\omega}$ such that, for each $\beta \in \omega_{1}, f_{\alpha}(\beta) \geq g(\beta)$. Let $V_{n}=U_{n} \cap\left\{f: f(\alpha) \in f_{\alpha}^{\prime \prime}\left(W_{n}\right)\right\}-\left\{d_{i}: i \leq n\right\}$. Claim that $\left\{V_{n}: n \in \omega\right\}$ is a locally finite family of open sets in $Y$ which contains the range of $\pi$. $\left\{f: f(\alpha)=f_{\alpha}(\beta)\right\}$ is a neighborhood of $\pi(\beta)$. Suppose $\left\{f: f(\alpha)=f_{\alpha}(\beta)\right\}$ intersects $V_{n} .\left\{f: f(\alpha)=f_{\alpha}(\beta)\right\}$ must intersect $\left\{f: f(\alpha) \in f_{\alpha}^{\prime \prime}\left(W_{n}\right)\right\}$ and so $f_{\alpha}(\beta) \in f_{\alpha}^{\prime \prime}\left(W_{n}\right)$. For each $\gamma \in \omega_{1}$, $f_{\alpha}(\gamma) \geq g(\gamma)$, for each $\gamma \in W_{n}, g(\gamma)=n$ and so $f_{\alpha}(\gamma) \geq n$. $\left\{f: f(\alpha)=f_{\alpha}(\beta)\right\}$ is a neighborhood of $\pi(\beta)$ which intersects $V_{n}$ for, at most, the finitely-many $n$ such that $n \leq f_{\alpha}(\beta) .\left\{d_{i}\right\}$ is a neighborhood of $d_{i}$ intersecting $V_{n}$ for, at most, the finitelymany $n \leq i$ and so we have shown that $\left\{V_{n}: n \in \omega\right\}$ is a locally finite family of open sets of $Y$ containing the range of $\pi .\left\{V_{n}: n \in \omega\right\} \cup\left\{\{d\}: d \in D-\bigcup\left\{V_{n}: n \in \omega\right\}\right\}$ is a locally finite refinement of $\left\{U_{n}: n \in \omega\right\}$ as required.

Question 2. Does $2^{\aleph_{0}}<2^{\aleph_{1}}$ imply that first countable separable countably paracompact spaces are collectionwise Hausdorff?

Question 3. Does $2^{\aleph_{0}}<2^{\aleph_{1}}$ imply that special Aronszajn trees are not countably paracompact? 
Question 4 . Let $X$ be a first countable countably paracompact space. Is it true that if the continuum function is one-one then $e(X) \leq c(X)$ ?

It is true if $X$ is a first countable normal space [12].

A weak form of countable paracompactness is used throughout this paper; it states that, whenever $\left\{A_{n}: n \in \omega\right\}$ are disjoint closed sets, there exists a locally finite open family $\left\{O_{n}: n \in \omega\right\}$ such that, for each $n \in \omega, O_{n}$ contains $A_{n}$ and is disjoint from $A_{m}$ for each $m \neq n$.

This weak form of countable paracompactness follows from normality and so Lemma 2 generalizes Lemma 1. This is not surprising when one notes that, if there is a first countable normal space which is not collectionwise Hausdorff, then there is such a space such that $\left(X^{\prime}\right)^{\prime}=\emptyset$ and that, in any regular space such that $\left(X^{\prime}\right)^{\prime}=\emptyset$, normal implies countably paracompact (where ' is the derived set operation).

An analog of countably paracompact is $\aleph_{1}$-paralindelöf (every open cover of cardinality $\aleph_{1}$ has a locally countable open refinement).

Fleissner [16] has shown that $V=L$ implies that first countable $\aleph_{1}$-paralindelöf spaces are collectionwise Hausdorff.

\section{BIBLIOGRAPHY}

1. W. G. Fleissner, Normal Moore spaces in the constructible universe, Proc. Amer. Math. Soc. 46 (1974), 294-298.

2. __ When is Jones' space normal?, Proc. Amer. Math. Soc. 50 (1975), 375-378.

3. __ Separation properties in Moore spaces, Fund. Math. 98 (1978), 279-286.

4. __ Remarks on Suslin properties and tree topologies, Proc. Amer. Math. Soc. 80 (1980), 320326.

5. R. Heath, Separation and $\aleph_{1}$-compactness, Colloq. Math. 12 (1964), 11-14.

6. T. Jech and K. Prikry, Cofinality of the partial ordering on functions from $\omega_{1}$ into $\omega$ under eventual domination, Math. Proc. Cambridge Philos. Soc. 95 (1984), 25-32.

7. F. B. Jones, Concerning normal and completely normal spaces, Bull. Amer. Math. Soc. 43 (1937), 671-677.

8. T. Przymusinski, Normality and separation in Moore spaces, Set-Theoretic Topology (G. M. Reed, ed.), Academic Press, New York, 1977, pp. 325-337.

9. G. M. Reed, On normality and countable paracompactness, Fund. Math. 110 (1980), 145-152.

10. J. Steprans, Some results in set theory, Thesis, University of Toronto, Toronto, 1982.

11. F. D. Tall, Set-theoretic consistency results and topological theorems concerning the normal Moore space conjecture and related problems (Thesis, Univ. of Wisconsin, Madison, 1969), Dissertationes Math. 148 (1977).

12. _ Weakly collectionwise Hausdorff spaces, Topology Proc. 1 (1975), 295-304.

13. M. E. Rudin, Lectures on set-theoretic topology, CBMS Regional Conf. Ser. in Math., no. 23, Amer. Math. Soc., Providence, R.I., 1975.

14. K. Kunen, Set theory, North-Holland, New York, 1980.

15. W. S. Watson, Locally compact normal spaces in the constructible universe, Canad. J. Math. 34 (1982), 1091-1096.

16. W. G. Fleissner, Discrete sets of singular cardinality, Proc. Amer. Math. Soc. 88 (1983), 743-745.

17. Z. Balogh, On countably paracompact, locally compact, submetacompact spaces (preprint).

18. P. Daniels, Manuscript.

19. D. Burke, PMEA and first countable, countably paracompact spaces, Proc. Amer. Math. Soc. 92 (1984), 455-460.

20. P. Nyikos, A provisional solution to the normal Moore space problem, Proc. Amer. Math. Soc. 78 (1980), 429-435.

Department of Mathematics, York University, Toronto, Ontario M3J 1P3, CANADA 